\title{
POPULATION STRUCTURE OF MORPHOLOGICAL TRAITS IN CLARKIA DUDLEYANA. II. CONSTANCY OF WITHIN-POPULATION GENETIC VARIANCE
}

\author{
Robert H. Podolsky, ${ }^{1,2}$ Ruth G. Shaw, ${ }^{1,3}$ and Frank H. Shaw ${ }^{3}$ \\ 'Department of Botany and Plant Sciences, University of California, Riverside, California 92521 \\ and ${ }^{2}$ Department of Biology, University of Michigan-Flint, Flint, Michigan 48502 \\ E-mail: robert@woodstar.fint.umich.edu \\ ${ }^{3}$ Department of Ecology, Evolution and Behavior, University of Minnesota, Saint Paul, Minnesota 55108 \\ E-mail: shaw@superb.ecology.umn.edu
}

\begin{abstract}
Recent quantitative genetic studies have attempted to infer long-term selection responsible for differences in observed phenotypes. These analyses are greatly simplified by the assumption that the within-population genetic variance remains constant through time and over space, or for the multivariate case, that the matrix of additive genetic variances and covariances ( $\mathbf{G}$ matrix) is constant. We examined differences in $\mathbf{G}$ matrices and the association of these differences with differences in multivariate means (Mahalanobis $D^{2}$ ) among 11 populations of the California endemic annual plant, Clarkia dudleyana. Based on nine continuous morphological traits, the relationship between Mahalanobis $\mathrm{D}^{2}$ and a distance measure summarizing differences in $\mathbf{G}$ matrices reflected no concomitant change in (co)variances with changes in means. Based on both broad- and narrow-sense analyses, we found little evidence that $\mathbf{G}$ matrices differed between populations. These results suggest that both the additive and nonadditive (co)variances for traits have remained relatively constant despite changes in means.
\end{abstract}

Key words.-Clarkia, constancy of $\mathbf{G}$ matrices, morphological evolution, quantitative genetics, population differentiation.

\author{
Received April 15, 1996. Accepted July 22, 1997.
}

Quantitative genetic analyses have become an integral part of efforts to understand the evolutionary processes responsible for observed changes in phenotypes. One application of quantitative genetics to evolution has been to examine phenotypic evolution in retrospect to assess whether observed phenotypic changes can be explained by genetic drift or by selection (Lande 1979; Price et al. 1984; Price and Grant 1985; Arnold 1988; Lofsvold 1988; Turelli et al. 1988). Such analyses are greatly simplified by the assumption, stemming from a Gaussian model of allelic effects (Lande 1979), that the within-population, additive-genetic variance remains constant, despite changes in means. In a multivariate sense, the additive-genetic, variance-covariance matrix (A matrix) is assumed to be constant. While retrospective analyses assume that the $\mathbf{A}$ matrix is constant through time, recent models of the geographic structure of quantitative traits also make this assumption over space (Lande 1991; Nagylaki 1994; but see Lande 1992).

Constancy of the A matrix is inconsistent with single-gene theory. For single genes, the variance depends directly on allele frequencies and thus changes as allele frequencies change (Falconer 1981). Constancy of $\mathbf{A}$ relies on the assumption that many loci, each of small effect, contribute to the overall variation in quantitative traits. If trait means change, the accompanying changes in allele frequencies are expected to have negligible effects on the genetic variance. However, quantitative traits whose variation is due to segregation at few loci are expected to show a change in withinpopulation variances and covariances with changes in means. Bohren et al. (1966) suggested that this will be especially true for the covariance.

Turelli (1988a) addressed the question of whether a constant $\mathbf{A}$ matrix is expected in natural populations, and pro-

\footnotetext{
2 Present address.
}

vided theoretical criteria for assessing the constancy of $\mathbf{A}$ matrices within models of selection-mutation balance. Barton and Turelli (1987) and Turelli (1988b) showed that when the effects of selection on allele frequencies are analyzed theoretically, changes in means are generally accompanied by changes in variance, and that the dynamics of the means are dependent on the dynamics of the variance. Turelli (1988a) concluded that we have too little data to judge the validity of the assumption of constant $\mathbf{A}$ matrices. The important point is that when populations differ in the $\mathbf{A}$ matrices, the subsequent evolution of those populations will differ under either genetic drift or selection. Further, when the $\mathbf{A}$ matrix differs between populations, it is not possible to infer the selection responsible for observed differences in means.

Tests of the constancy assumption have followed two main approaches. The first examines the correlated response to selection. In such experiments, the observed correlated response of one trait to selection on another (realized covariation) is compared between subsequent generations exposed to selection (e.g., Falconer 1960), or between replicate selection lines (e.g., Bell and McNary 1963). These studies have generally shown that the genetic covariance differs between subsequent generations or replicate selection lines providing evidence that genetic covariances are not constant. Hoffman and Cohan (1987) also found different correlated responses of populations of Drosophila pseudoobscura. Bohren et al. (1966) showed that, when few loci underlie the traits, discrepancies between the realized covariation of different populations or subsequent generations are expected to be common. Further, predictions of correlated response are likely to be accurate for a much shorter time than for direct response. Gromko (1995) suggests that differences in correlated responses among replicate populations might be due to variability of pleiotropic effects among loci. In this scenario, genes with differing pleiotropic effects are fixed in 
different populations. Similarly, Heath et al. (1995) have shown that both upward and downward selection on body weight changes both the genetic and environmental variances. Further, the changes observed were inconsistent with an infinitesimal model of allelic effects. The implications for quantitative genetic population structure are not clear.

The second type of experiment investigating the constancy of $\mathbf{A}$ matrices has compared estimates of the $\mathbf{A}$ matrices between populations (Billington et al. 1988; Wilkinson et al. 1990; Shaw and Billington 1991) or taxa (Lofsvold 1986; Paulsen 1996). Some have compared populations or taxa by examining broad-sense estimates of the genetic (co)variance matrix (G matrix; Kohn and Atchley 1988; Platenkamp and Shaw 1992; Brodie 1993). Platenkamp and Shaw (1992) and Brodie (1993) found no significant differences between G matrices of populations of Anthoxanthum odoratum, a grass, and Thamnophis ordinoides, a garter snake, respectively. Paulsen (1996) also found that two species of butterfly, Precis coenia and $P$. evarete, had $\mathbf{G}$ matrices that did not differ significantly. Billington et al. (1988) provided evidence of differences in A matrices among populations (Shaw and Billington 1991). These comparisons of distinct, contemporary populations are often used to make inferences about the constancy of $\mathbf{A}$ matrices through time. The underlying assumption of such comparisons is that the populations have arisen from some common ancestral population, and that the two populations reflect the original $\mathbf{A}$ matrix and changes in it through time.

Wilkinson et al. (1990) conducted an experiment that combines features of both of the approaches mentioned above. Comparison of $\mathbf{A}$ matrices between two selected lines and a base population of Drosophila melanogaster revealed significant differences between the base population and the line selected for small thorax length but not between the base population and the line selected for large thorax length, as shown by a reanalysis of these data (Shaw et al. 1995). The A matrices differed significantly between these two selected lines, suggesting that selection can alter the $\mathbf{A}$ matrix. A great strength of the Wilkinson et al. (1990) study is that changes in means can be directly associated with changes in (co)variance.

An inherent difficulty in comparing $\mathbf{A}$ matrices between populations is that, because the number of parameters to be estimated is often large statistical tests demand unusually large sample sizes for a given level of power (Shaw 1991). A further approach to investigating the constancy of $\mathbf{A}$ matrices follows from the findings of Turelli (1988b) and Barton and Turelli (1987) that changes in means will be accompanied by changes in the $\mathbf{A}$ matrix, under some conditions. Thus, one expectation is to find a relationship between differences in $\mathbf{A}$ matrices and differences in the means. Such a finding would provide evidence that $\mathbf{A}$ matrices are not constant, even if no direct comparison of two populations' $\mathbf{A}$ matrices reveals significant differences.

In this paper, we will examine how genetic (co)variance matrices differ among populations in relation to the degree of genetic differentiation among them by asking the following two questions for morphological traits. Does differentiation of $\mathbf{G}$ matrices increase with differences in means? If so, how great must the differentiation of means be for detection of significant differences in $\mathbf{G}$ matrices? To address these questions, we present studies of the California endemic wildflower, Clarkia dudleyana. Lewis (1962) and Lewis and Raven (1958) have suggested that population structure has been important in the evolution of the genus Clarkia, as a whole, by proposing that peripheral populations allow for the rapid speciation observed in Clarkia. Because speciation has been rapid in this genus, differences in the genetic (co)variance structure might be expected. We have been examining aspects of the quantitative genetic structure of $C$. dudleyana as a basis for understanding morphological evolution in this genus. Previously, Podolsky and Holtsford (1995) showed that the population structure of some quantitative traits differ from that of allozymes. Further, the quantitative traits differed in the degree of population genetic subdivision. We examine the structuring of the genetic (co)variance matrices in relation to the extent of genetic differentiation in this species. To answer these questions for a collection of 11 populations, we use broad-sense quantitative genetic analysis. We also directly compare A matrices, estimated in the narrow sense, for two of the populations.

\section{Materials ANd Methods}

Clarkia dudleyana (Abrams) J. F. Macbr. (Onagraceae) is an annual endemic to California. Populations in the Sierra Nevada foothills cover large areas and are continuous, whereas populations in southern California are smaller and more discrete (Podolsky, pers. obs.) as they have been for at least 45 years (Lewis and Lewis 1955). The southern California populations range in size from a few to thousands of individuals. Clarkia dudleyana is a self-compatible, outcrossing species that germinates between November and January and flowers between May and August.

We sampled much of the range of $C$. dudleyana in southern California (Podolsky and Holtsford 1995), collecting capsules by maternal plant from 11 populations (Fig. 1, Appendix Table A1). Details of sampling can be found in Podolsky and Holtsford (1995).

\section{Broad-Sense Experiment}

We germinated seed from 20 sibships per population using the protocol in Podolsky and Holtsford (1995). Briefly, at weekly intervals at least two seeds per sibship were randomly selected, germinated, and planted in a lathhouse at the University of California, Riverside. Plants in pots were thinned to a single plant, and any pots missing seedlings two weeks later were replanted. Overall survivorship to flowering was 94.4\%. Clarkia dudleyana is an outcrossing species, therefore the five plants per sibship will represent a mixture of both full- and half-sibs. We assumed that these plants represented a maternal full-sib family since this assumption provides conservative estimates of the quantitative genetic parameters. We measured both continuous and discrete morphological traits (Table 1). These traits were chosen as measures of the observable morphological variation, without any knowledge of selection affecting these traits. Presence or absence of leaf spots (LS) was scored four weeks after planting. The remaining traits were measured on the first day of flowering. Tests of normality for all traits were conducted on residuals 


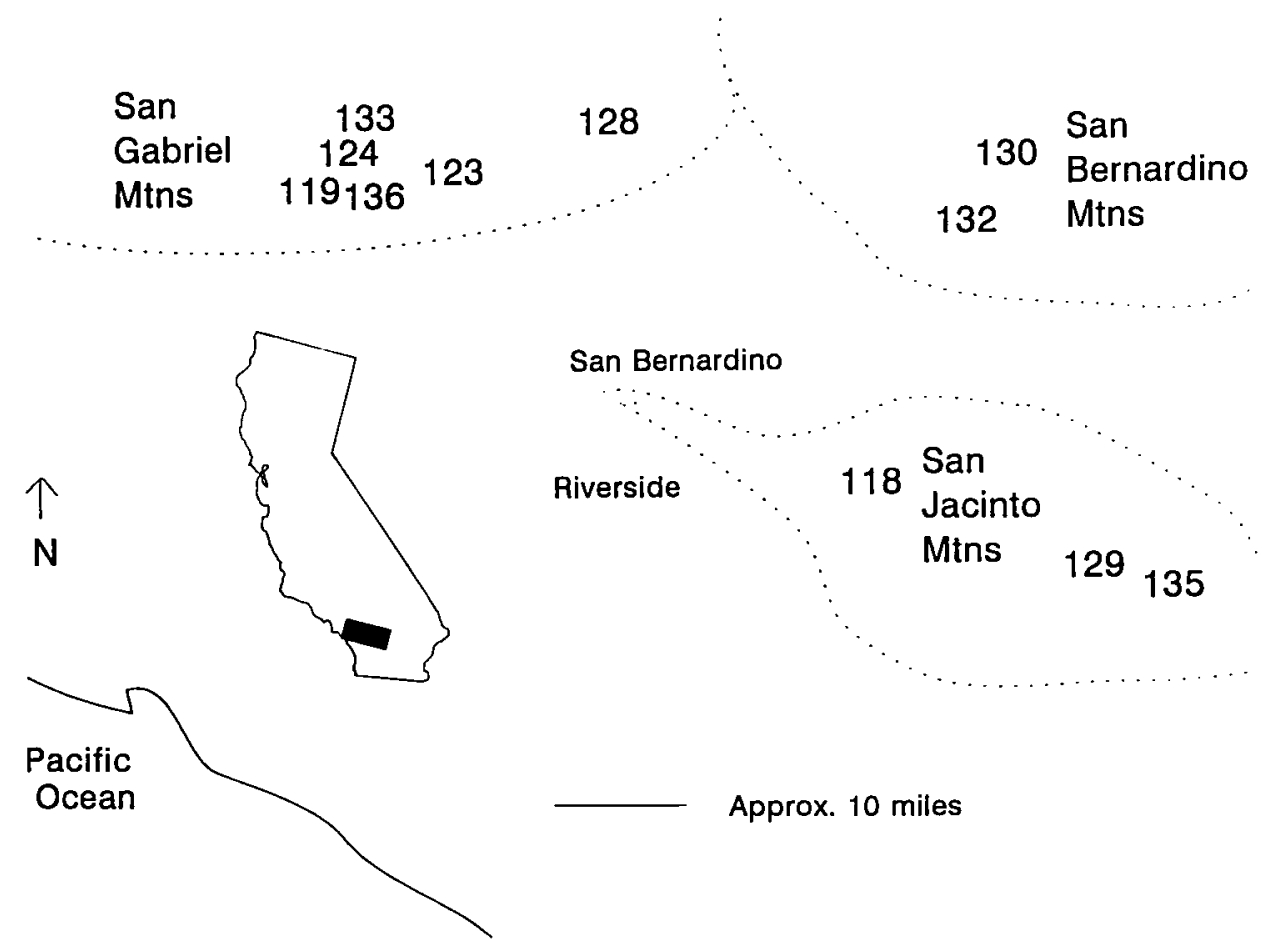

FIG. 1. Map of the populations from which seed was collected. The shaded box in the inset map of California shows the approximate location of the larger map. See Appendix Table A1 for details of collection sites.

obtained from a nested analysis of variance with maternal family nested within population using the procedure Proc Univariate in SAS (SAS Institute 1985). The traits were transformed to normality as feasible (Table 1). As transformed, all of the continuous traits were normally distributed. The trait dark band (DB) had a marginally significant deviation from normality $(P=0.0406)$ and no transformation improved the fit to normality. Therefore, DB was left untransformed in all analyses. Although the residuals for the transformed discrete traits did not fit a normal distribution, we used the transformation that most closely fit a normal distribution.

We used restricted maximum likelihood (REML; Shaw 1987 ) in which the (co)variance components are constrained to feasible estimates (all variances $\geq$ zero; all correlations in the range, $-1 \leq r \leq 1$; Shaw and Geyer 1997) to estimate and compare $\mathbf{G}$ matrices between the populations using fullsib families (Shaw 1991; Shaw and Shaw 1992). Estimates of the genetic variance obtained from this design include fractions of components other than the additive variance: dominance variance, $V_{D}$; variance due to maternal effects, $\mathrm{V}_{\mathrm{M}}$; and variance due to epistasis, $\mathrm{V}_{\mathrm{I}}$. We conducted separate analyses for the continuous and discrete traits.

A comparison of all populations in a pairwise fashion would require 55 tests, which is prohibitive in terms of computational time required. To reduce the number of pairwise comparisons of $\mathbf{G}$, we systematically compared populations based on decreasing morphological similarity as follows. We calculated Mahalanobis $\mathrm{D}^{2}$, a multivariate measure of morphological distance based on means, between all pairs of populations using the procedure Proc Candisc in SAS (SAS Institute 1985). These distances were then organized into a distance matrix. We used the distance matrices to compute a
UPGMA clustering tree (Sneath and Sokal 1973) for the discrete and continuous traits using Felsenstein's (1989) PHYLIP. The resulting trees were used to choose pairs of populations to compare, beginning with the pair that differed least and ending with a pair differing most with respect to means. The first comparison involved the two populations connected by the shortest branch of the UPGMA tree. The next comparison included the populations connected by the next shortest branch. If this branch connected a pair of populations with a single population, then the closest of the pair to the single population was chosen for the comparison. If a branch connected pairs of populations, then the closest population from one pair to the closest of the other would then be compared. We provide an example in Figure 2.

Given the relatively small sample sizes within populations, the entire $\mathbf{G}$ matrix for either the continuous or discrete traits cannot be compared between any two populations; we have too few observations to estimate the many parameters simultaneously (Shaw 1987, 1991). We therefore included only the traits determined to have most power for discriminating between the means of the two populations under consideration using Akaike's information criterion in a discriminant function analysis framework (Fujikoshi 1985; Podolsky, unpubl.). Using these informative traits in such a manner is equivalent to focusing on the traits that differ most in mean value and for which we would therefore predict most change in genetic variances under an oligogenic model. We expect this procedure to provide relatively great power to detect differences in $\mathbf{G}$ matrices. Using this procedure, each specific comparison is based on a subset of the traits that is likely to differ from other comparisons. Still, too many traits were determined to be informative for a single analysis for many 
TABLE 1. Morphological traits measured.

Continuous morphological traits

LL: length of longest leaf

LW: width of longest leaf

IL1: distance between last pair of opposite branches and first alternate branch (long internode length); transformed as $Y$ $=\sqrt{X}$ for the broad-sense analyses and $\mathrm{Y}=\ln (\ln [\mathrm{X}]+1)$ for the narrow-sense analyses

IL2: distance between first two alternate branches (short internode length); transformed as $\mathrm{Y}=\ln (\ln [\mathrm{X}]+1)$ for the broadsense analyses and $\mathrm{Y}=\ln (\mathrm{X})$ for the narrow-sense analyses

PL: length of petal on first flower

PW: petal width of first flower; transformed as $Y=\sqrt{X}$ for the broad-sense analyses and not transformed for the narrowsense analyses

CW: width of the constricted petal base (claw); transformed as $\mathrm{Y}=\sqrt{\mathrm{X}}$ for the broad-sense analyses and $\mathrm{Y}=\ln (\mathrm{X})$ for the narrow-sense analyses

OL: ovary length

HL: hypanthium length

Discrete morphological traits

LS: presence or absence of leaf spots; transformed as $Y=\sqrt{X}$ $+\sqrt{(X+1)}$ for the broad-sense analyses and not measured in the narrow-sense analyses

PS: presence or absence of petal specks $(0=$ no specks, $1=$ few specks, 2 = many specks)

WS: white spot present or absent from cup of petal $(0=$ no spot, $1=$ small spot, $2=$ large spot) ${ }^{2}$

DB: dark band separating claw and petal present or absent ${ }^{2}(0=$ no band, 1 = light band, 2 = dark band)

WC: color of the claw is either white or pink ${ }^{1}(0=$ dark pink claw, 1 = light pink claw, 2 = white claw)

RB: the ovary is either curved (1) or straight (0) at anthesis 1

SC: color of the nonreceptive stigma is either white (0), light pink (1), or dark pink (2) ${ }^{2}$

St: presence (1) or absence $(0)$ of white streaks on the petals ${ }^{1}$

Pub: the stem is either pubescent $(1=$ pubescent, $2=$ very pubescent) or glabrous $(0)^{1}$

${ }^{1}$ Traits transformed as $Y=\sqrt{(X+1)}$ for the broad-sense analyses and $Y$ $=\sqrt{X}+\sqrt{(X+1)}$ for the narrow-sense analyses.

${ }^{2}$ Traits transformed as $Y=\sqrt{X}+\sqrt{(X+1)}$ for the narrow-sense analyses and not transformed for the broad-sense analyses.

of the discrete trait comparisons. We therefore compared multiple submatrices of $\mathbf{G}$, making sure to include all (co)variances in at least one submatrix comparison. The populations compared and the traits analyzed for each comparison are presented in Table 2.

Pairs of $\mathbf{G}$ matrices were compared using the Quercus programs of Shaw and Shaw (1992). The likelihood ratio test (LRT) was subsequently calculated, and tested against a chisquare distribution with the appropriate degrees of freedom. This test statistic is not expected to have an exact chi-square distribution when feasibility constraints are used. Shaw and Geyer (1997) provide a method, the asymptotic parametric bootstrap (APB), for conducting tests with such constraints in place. We initially calculated the LRT statistic and then used the APB when the LRT approached significance.

Although the discrete traits cannot be considered normally distributed data, we used the methods described above in the absence of detailed information about the distributions. The methods for estimating variance components of discrete data are just beginning to be developed (e.g., McCulloch 1994), so we analyzed the discrete traits in the same manner as the

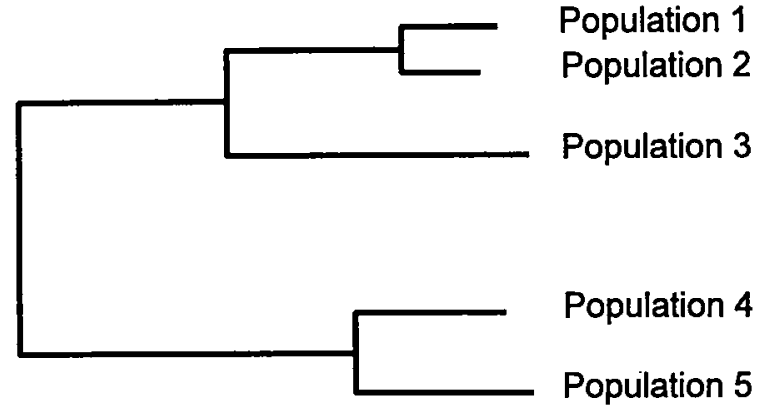

Fig. 2. An example of a UPGMA clustering tree used as a basis for comparing populations. Based on the tree in this figure, we would first compare populations 1 and 2 . The next comparison would involve populations 4 and 5 . The next comparison would include populations 2 and 3 , assuming 2 is closer to 3 than is 1 . The final comparison would involve populations 2 and 4 , assuming the distance between 2 and 4 is the shortest of the distances between populations 2 and 4,2 and 5,3 and 4 , and 3 and 5 . See text for further details.

continuous traits. The assumptions inherent in the analyses, that these traits are influenced by many loci, may be violated for these traits, and the $P$-values should be viewed cautiously.

To examine the qualitative relationship between differences in means and differences in $\mathbf{G}$, we first calculated a "distance" measure for two $\mathbf{G}$ matrices $\left(\mathrm{GD}^{2}\right)$ as follows. The differences in corresponding elements of the two estimated matrices were squared and summed over all elements in a pairwise manner. This sum of squared differences was then divided by the product of the number of (co)variances (elements of a single matrix) and the average value for the elements of the matrices. The average value for the elements was used as a denominator to standardize the mean squared

TABLE 2. Populations compared and traits analyzed for the broadsense analyses.

\begin{tabular}{ll}
\hline \hline Populations & \multicolumn{1}{c}{ Traits } \\
\hline 123,136 & LL, IL1, CW, OL \\
124,130 & LL, IL1, PL, PW, HL \\
119,132 & PW, OL, HL \\
129,135 & LW, IL1, PL, CW, OL, HL \\
124,132 & LW, PW, CW, OL, HL \\
118,129 & IL1, PL, CW, HL \\
124,136 & LL, LW, IL1, PW, CW \\
118,133 & LL, LW, IL1, IL2, CW, OL \\
118,124 & IL1, IL2, PL, HL \\
124,128 & LL, PL, PW, OL \\
118,129 & WS, St, Pub \\
123,128 & LS, RB, Pub \\
129,135 & LS, WS, WC, SC, Pub \\
123,133 & PS, WC, RB, SC, St, Pub \\
119,136 & PS, WC, SC, Pub \\
130,132 & LS, PS, WS, DB, WC, RB, Pub \\
119,123 & PS, WS, DB, SC \\
119,124 & LS, PS, WS, WC, RB, SC \\
124,135 & LS, PS DB, WC, RB, SC, St, Pub \\
132,135 & LS, PS, WS, DB, SC, Pub \\
$119,130^{*}$ & LS, PS, WS, DB, WC, SC, St, Pub \\
$119,132 *$ & LS, PS, WS, WC, SC, Pub \\
$124,132^{*}$ & LS, PS, WS, DB, WC, RB, St, Pub \\
\hline
\end{tabular}

* These additional pairs of populations were compared for the discrete traits to examine populations in different mountain ranges. 
distances, because different traits are included in different comparisons. As a result of scale differences between traits, the mean squared differences between matrices vary. $G^{2}$ was regressed against Mahalanobis $\mathrm{D}^{2}$ for both the discrete and continuous morphological traits. For the discrete traits, the average $\mathrm{GD}^{2}$ for a given comparison between two populations was used for the regression. Because this procedure is an ad hoc approach to examining the relationship between differences in $\mathbf{G}$ matrices and differences in means, we also calculated an alternate $\mathrm{GD}^{2}$ by dividing the squared difference of any pair of elements by the average of the elements, and taking the average of the standardized squared differences. We do not present these results here, since similar results were obtained with this alternate $\mathrm{GD}^{2}$.

\section{Narrow-Sense Experiment}

We conducted a set of controlled crosses within each of two relatively large populations occurring in distinct mountain ranges (populations 124 and 130, Fig. 1). Plants from the two populations were grown concurrently and randomly located throughout a greenhouse. We implemented a nested mating design (North Carolina I, Comstock and Robinson 1948 ) in which plants designated as paternal (sire) are mated to each of three maternal plants (dams). There were 495 plants available to serve as dams ( 300 from population 124 , and 195 from population 130 ) and 165 as sires (100 from population 124, and 65 from population 130). The final total number of maternal full-sib families produced from these crosses was 433 (276 from population 124, and 171 from population 130). The resulting sibships represent full-sib and paternal half-sib families. The procedural details of the matings are provided in Podolsky and Holtsford (1995). Briefly, flowers on plants designated as dams were emasculated and pollinated with anthers freshly collected from plants designated as sires. Control emasculations in which no pollen was deposited manually showed very little contamination, with only two of 44 capsules setting any seed and only five seeds total being produced from these capsules. A typical capsule in the greenhouse produces a minimum of 60 seeds. On this basis, the contamination rate for crosses was likely to be less than $0.2 \%$.

Progeny seed from these crosses were germinated such that a single plant per maternal full-sib family was grown in each of four blocks using the same planting protocol as in the broad-sense experiment. Plants from different populations were intermixed and randomly placed. The first three blocks were grown in a lathhouse at the University of California, Riverside. Excessive rain caused high mortality in these blocks. For this reason, an additional block was later grown in a greenhouse at the University of California, Riverside. Leaf spot (LS) was not scored for these progeny, but the remaining traits were measured on the first day of flowering. Tests of normality were conducted for all traits using residuals obtained from a nested analysis of variance (sire nested within population and dam nested within both sire and population), and the residuals were tested for normality using the procedure Proc Univariate in SAS (SAS Institute 1985). The traits were transformed to normality as feasible (Table 1). Following transformation, all the continuous traits were distributed normally. However, even after transformation, none of the discrete traits were normally distributed. The discrete traits were transformed as $Y=\sqrt{X}+\sqrt{(X+1)}$, which is expected to stabilize variances (Freeman and Tukey 1950).

The A matrices were estimated and compared using the Quercus programs of Shaw and Shaw (1992) with the feasibility constraints imposed. The mating design we used results in the dominance variance being confounded with maternal effects (Falconer 1981). For this reason, we considered alternative models, one in which one-quarter of the dominance variance contributes to the resemblance of full-sibs, and the other, with maternal variance contributing to full-sib resemblance. Again, we conducted these analyses with subsets of traits because the full comparison would have entailed estimation of more parameters than possible given our data. For the continuous morphological traits, we analyzed two subsets of the A matrix: vegetative and floral traits. We analyzed two subsets for the discrete morphological traits, in which the first subset was the first four traits listed in Table 1 , excluding LS, and the second subset was the next four.

\section{RESUlts}

\section{Broad-Sense Experiment}

The means for all traits differed among the populations as determined by nested univariate analyses of variance $(P<$ 0.005 for all traits; means for the continuous traits and the frequencies for the discrete traits for each population are presented in the Appendix; Tables A2 and A3) with the exception of short-internode length (IL2). Taking all traits into account, some populations were very similar morphologically (e.g., populations 123 and 136 for the continuous traits; Table 3), while others were quite distinct as determined by Mahalanobis $\mathrm{D}^{2}$ (e.g., populations 124 and 128 for the continuous traits; Table 3). UPGMA clustering that was based on the matrices of Mahalanobis $\mathrm{D}^{2}$ differed between the discrete and continuous traits (Fig. 3 ). The clustering based on the discrete traits showed a strong geographical association with populations within each mountain range clustering together (Fig. 3). The clustering based on the continuous traits did not show this geographic association of the three mountain ranges.

None of the pairs of $\mathbf{G}$ matrices for the 10 population comparisons suggested by the UPGMA trees for either the continuous or the discrete traits differed significantly $(P>$ 0.05 for all comparisons). Because the UPGMA clustering analysis for the discrete traits reflects the geographic distribution of the three mountain ranges, most of the comparisons based on the tree are within-mountain range comparisons. For this reason, we randomly chose three additional pairs of populations to compare such that in each pair the two populations occur in different mountain ranges (Table 2). As was the case for the other population comparisons, we only examined those traits that proved to be informative using Akaike's information criterion. The comparison of the discrete trait $\mathbf{G}$ matrices between populations 119 and 130 showed significant differences for two submatrices: one that included the traits LS, petal specks (PS), white petal spot (WS), DB, and white claw (WC; LRT $=11.783 ; P=0.008$ ), and the 
TABle 3. Distance matrices between populations based on Mahalanobis $D^{2}$. Values for the continuous traits are listed on the first line and values for the discrete traits are listed on the second line of each cell.

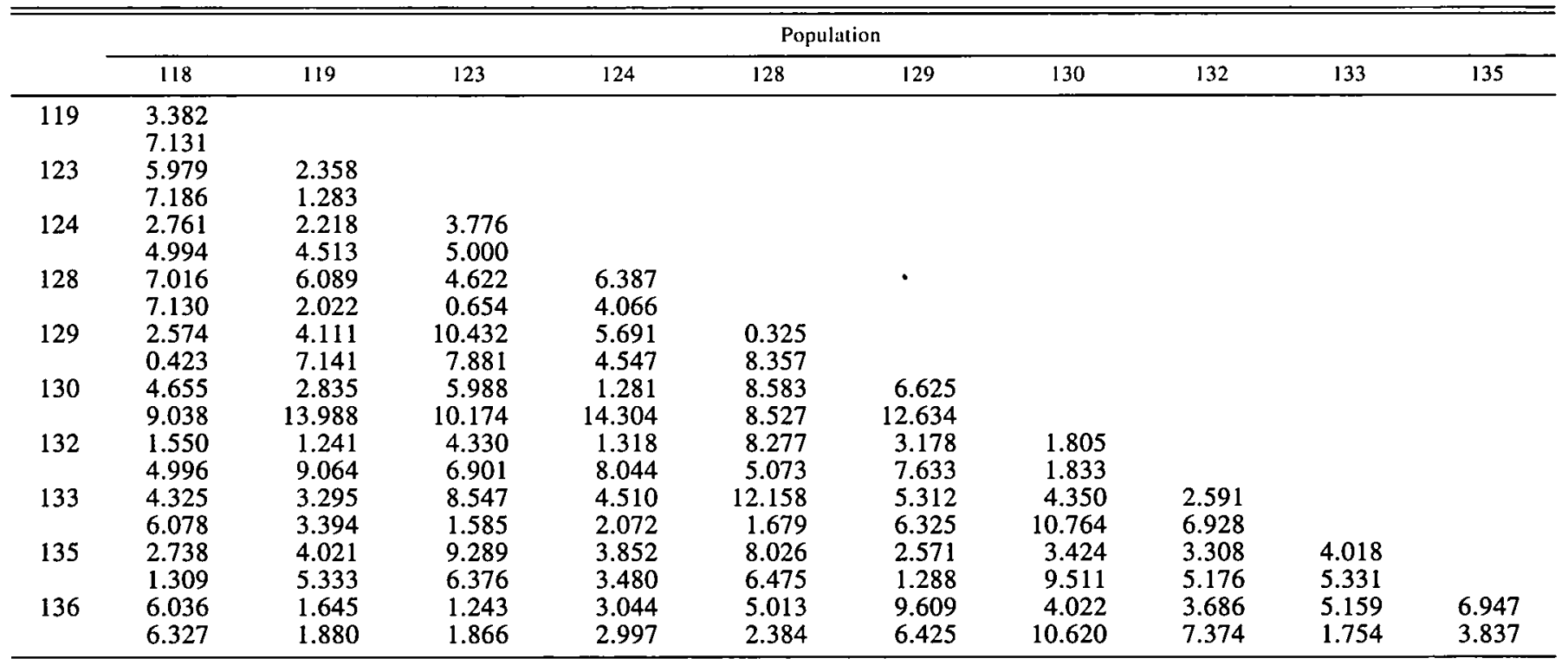

other included the traits LS, PS, WS, stigma color (SC), and petal streaks $(\mathrm{St} ; \mathrm{LRT}=11.893 ; P=0.016)$. The relationship between the $\mathrm{GD}^{2}$ and Mahalanobis $\mathrm{D}^{2}$ was positive, but not significant, for the discrete traits $(r=0.158 ; P=0.625$; Fig. 4) and was insignificantly negative for the continuous traits $(r=-0.150 ; P=0.679$; Fig. 4 ).

\section{Narrow-Sense Experiment}

The means for all of the continuous traits differed significantly between populations 124 and $130(P<0.01$ for all traits) except for IL2. The frequencies of the discrete traits WC and SC did not differ significantly, but the remaining discrete traits did $(P<0.005$ for all remaining traits). The matrices of the additive genetic variances and covariances (A) differed significantly from 0 for some traits, but not necessarily in both populations (Table 4). For example, the A matrix for the continuous floral traits for population 124 differed significantly from $\mathbf{0}$, but not for population 130 . The additive-genetic (A), dominance (D), and environmental (E) variance components all exhibited differences between the two populations for the continuous floral traits (Table 5), with the difference in A being statistically nonsignificant (LRT = 10.7014 , df $=15, P>0.05$ ). Likewise, the continuous vegetative and the discrete traits did not show a significant difference in A matrices.

To better compare the results between the broad- and narrow-sense experiments, we used a broad-sense analysis of the data from the formal crossing experiment to compare populations 124 and 130. Again, no significant difference in G matrices was found (for the continuous floral traits, LRT $=14.327, \mathrm{df}=15, P>0.05$ ). Further, the nonadditive genetic (co)variance matrix did not differ significantly from 0 using either the dominance (for the continuous floral traits, LRT $=10.164, \mathrm{df}=30, P>0.05$ ) or the maternal effects (for the continuous floral traits, LRT $=10.2216$, df $=30, P$ $>0.05$ ) for most comparisons. However, the $D$ matrix based on four discrete traits, reflexed bud (RB), SC, St, and pubescence (Pub), in population 124 differed significantly from $0($ LRT $=17.590, \mathrm{df}=15, P=0.001)$.

\section{Discussion}

When compared in the broad sense, very few significant differences in $\mathbf{G}$ matrices were observed. Further, this study revealed no consistent relationship between the measure of distances between $\mathbf{G}\left(\mathrm{GD}^{2}\right)$ and the measure of distances between means (Mahalanobis $\mathrm{D}^{2}$ ). These results provide little evidence for changes in (co)variances through evolutionary time or over space. Likewise, our experiment involving formal crosses in two populations yielded no clear indication of differences in genetic (co)variance matrices for either the continuous or discrete traits, regardless of whether the results were analyzed in the narrow or broad sense.

One potential explanation for the finding of no significant differences between populations compared in either the broad or narrow sense is the limitation in the power for any of four reasons. First, small sample size seriously restricts the statistical power of the $\mathbf{G}$ matrix comparisons (Shaw 1991). The much greater sample sizes per population used in the crossing experiment relative to those used in the survey of all 11 populations were nevertheless not sufficient to demonstrate differences in G. Second, power for comparing $\mathbf{G}$ matrices is known to decrease as the number of traits in the analysis increases. To determine whether we could detect differences in $\mathbf{G}$ by drastically reducing the number of traits, we focused on two traits, petal width (PW) and long-internode length (IL1), which exhibit relatively high levels of population divergence compared with the other continuous traits (Podolsky and Holtsford 1995). In tests of equality of A matrices using PW or IL1 singly, or with one other trait from the narrowsense experiment, we found no significant differences between populations 124 and 130 . Third, statistical power depends on the magnitude of the real differences. If the actual 


\section{Continuous Traits}

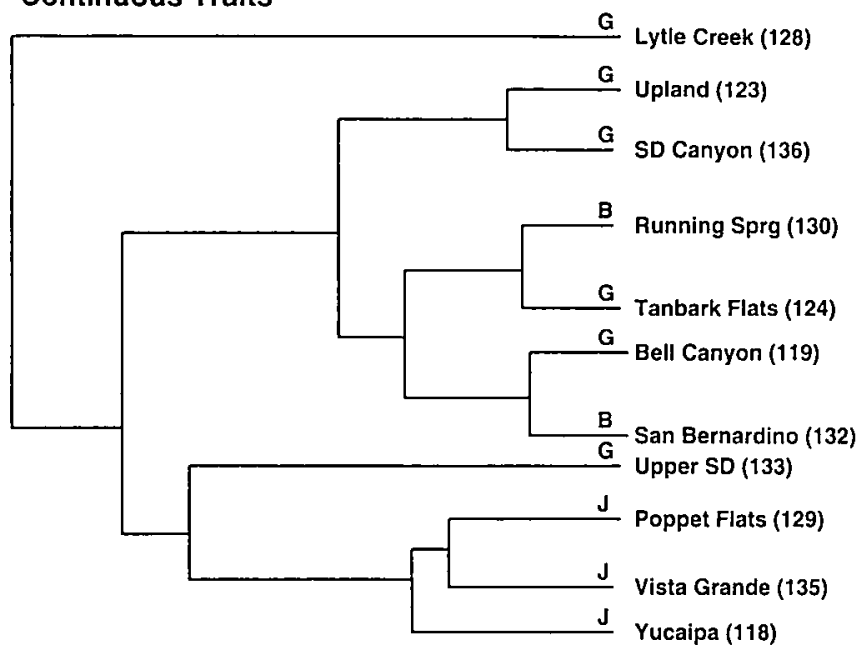

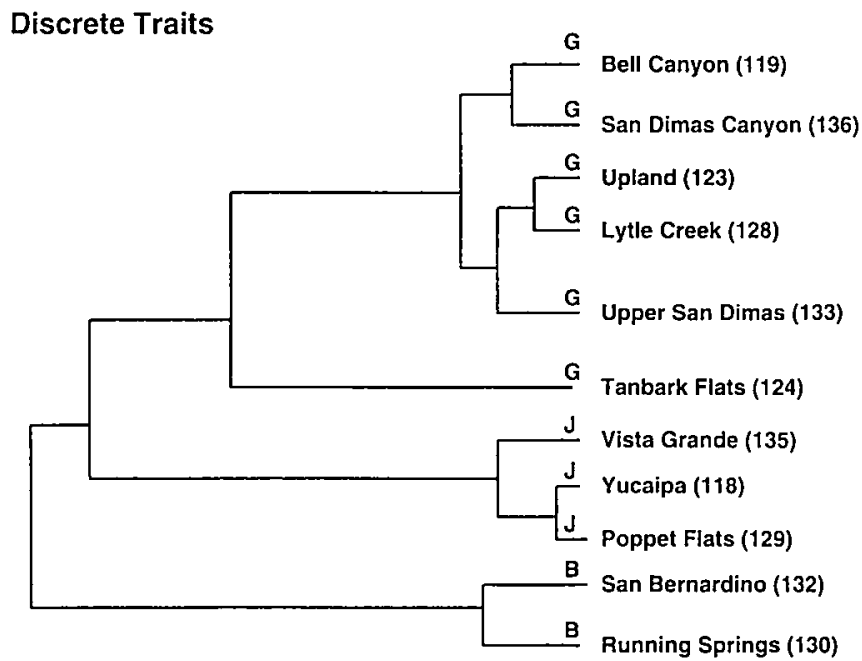

FIG. 3. UPGMA clustering trees for the morphological traits measured. The letters on the branches represent the mountain ranges to which the population belong: $B=$ San Bernardino; $G=$ San Gabriel; J = San Jacinto.

differences in $\mathbf{A}$ are small, then the size of the experiment required to detect such differences would make the experiment impractical. However, inspection of the A matrices for populations 124 and 130 indicates some apparently substantial differences (e.g., $\mathrm{V}_{\mathrm{A}}$ for petal length and PW; Table 5). Thus, this issue appears to be a less likely basis for our findings. Fourth, the sampling variances of the genetic (co)variances might be very large for traits with low heritabilities (Falconer and Mackay 1996). Many of the traits that differ between populations 124 and 130 exhibit a relatively small heritability in one of the populations (e.g., $h^{2} \mathrm{PW}$ for population $124=4.27 \%$ and $h^{2} \mathrm{pw}$ for population $130=$ $19.74 \%$; Table 5). Inordinately large families would be required to reduce the sampling variance for traits with low heritabilities. In the present study, this would make it difficult to detect differences due to the low heritabilities in one of the populations.

We also attempted to examine the qualitative relationship
Continuous Traits

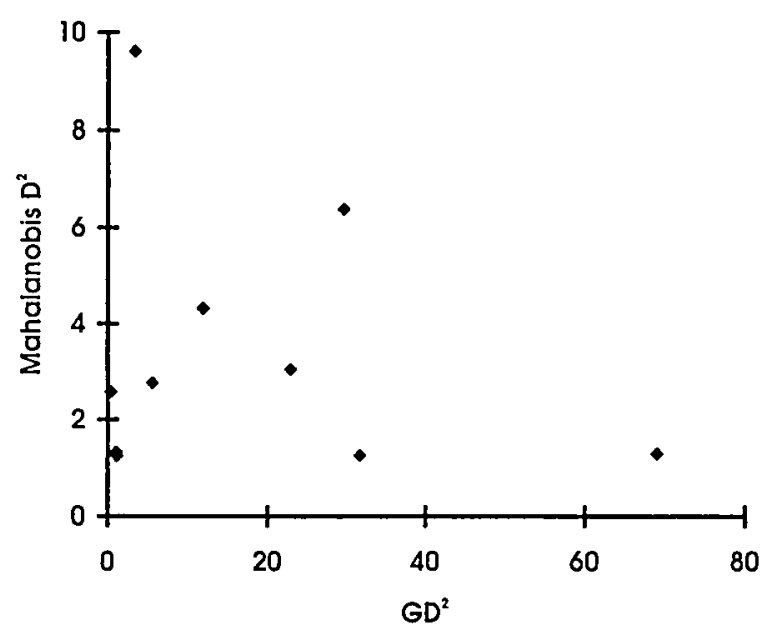

Discrete Traits

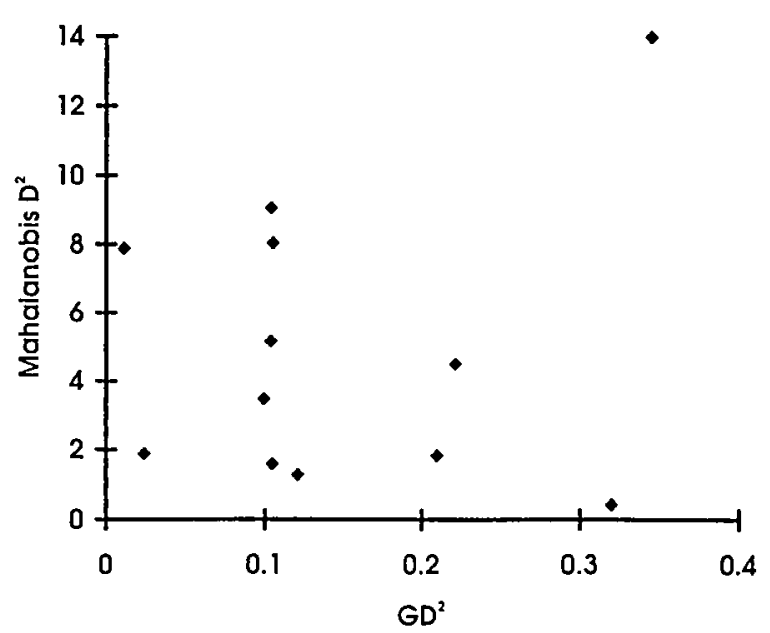

FIG. 4. Relationship between a distance measure for two $\mathbf{G}$ ma trices and a distance measure for means. $\mathrm{GD}^{2}$ is calculated as noted in the results, and is the measure based on differences in $\mathbf{G}$ matrices. Mahalanobis $\mathrm{D}^{2}$ represents the distance for means.

TABLE 4. Tests for the additive genetic (co)variance matrix equal to 0 for both populations 124 and 130 . The $P$-value given is approximate. NS $=$ not significant.

\begin{tabular}{lcrll}
\hline \hline \multicolumn{1}{c}{ Traits } & $\begin{array}{c}\text { Popula- } \\
\text { tion }\end{array}$ & \multicolumn{1}{c}{ LRT } & df & \multicolumn{1}{c}{$P$-value } \\
\hline LL, LW, IL1, IL2 & 124 & 8.3816 & 10 & NS \\
& 130 & 17.2712 & 10 & $0.05<P<0.10$ \\
PL, PW, CW, & 124 & 60.7012 & 15 & $P<0.001$ \\
OL, HL & 130 & 15.3694 & 15 & NS \\
PS, WS, DB, WC & 124 & 18.0902 & 10 & $P=0.05$ \\
& 130 & 19.4448 & 10 & $0.025<P<0.05$ \\
RB, SC, St, Pub & 124 & 14.1444 & 10 & NS \\
& 130 & 15.3818 & 10 & NS \\
\hline
\end{tabular}


TABLE 5. The additive-genetic, dominance, and environmental (co)variance matrices for continuous floral traits measured on plants from the populations 124 and 130 estimated in the narrow sense. The matrices for the other traits exhibited similar differences between populations.

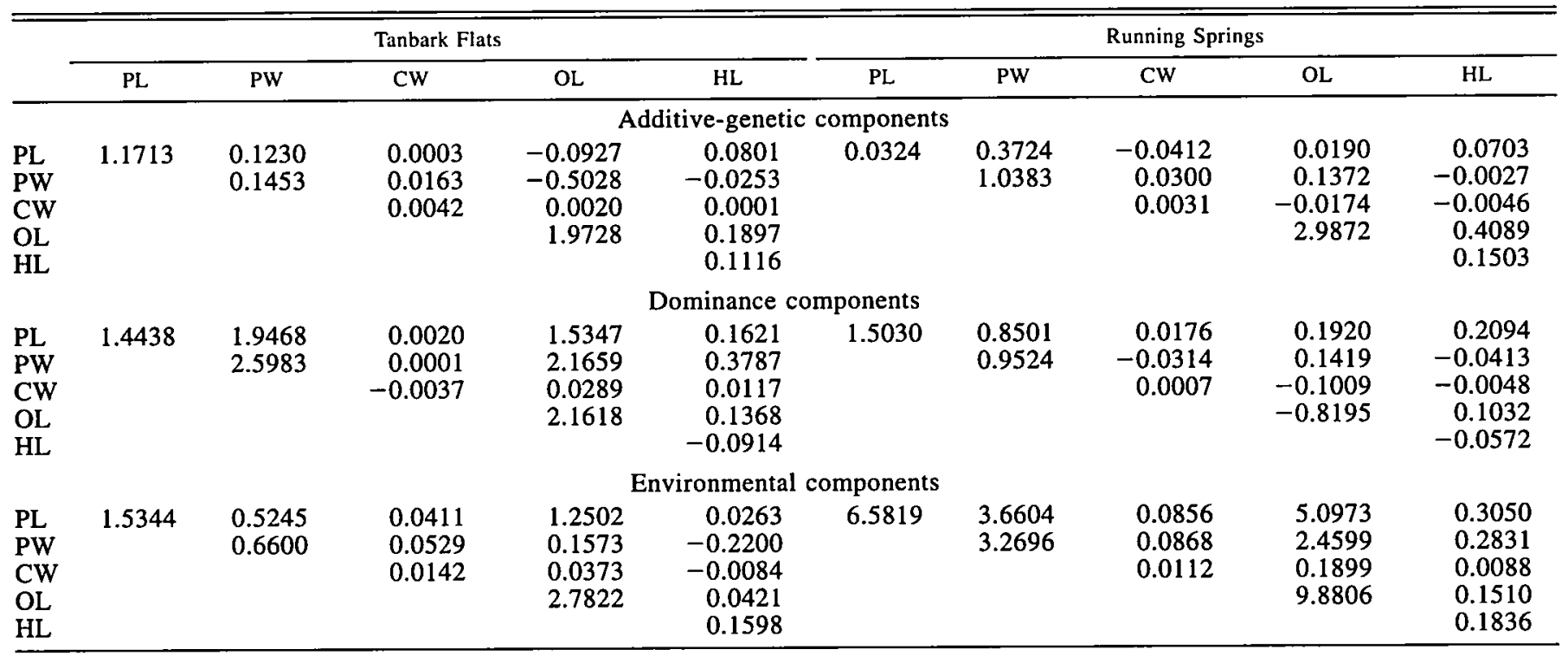

between differences in the $\mathbf{G}$ matrices and differences in means as a way of examining the constancy of quantitative genetic variation that is not as affected by the power of the test statistics used here. The finding of no relationship between differences in $\mathbf{G}$ and differences in means does not necessarily imply that $\mathbf{G}$ matrices are constant. Barton and Turelli (1987) do provide some theoretical foundation for expecting a monotonic relationship between the differences in variances and the differences in means, but they note that their theory should not be used to make quantitative predictions.

Wilkinson et al. (1990) examined the effects of selection on changes in means and $\mathbf{A}$. In that study, populations of $D$. melanogaster were strongly selected for either large or small thorax length for 23 generations. The $\mathbf{A}$ matrices for five morphological traits were then compared between these two populations, a control population and the base population from which the other three populations were derived. The population selected for small thorax length showed greater response in the selected trait than did the one selected for large thorax length. In addition, more of the morphological trait means differed between the small thorax population and the control or base than did the large thorax population. Accompanying an asymmetrical response to selection was an asymmetrical change in $\mathbf{A}$ matrices where the small-thorax population differed more from the base population than did the large-thorax population. Although an asymmetrical change in A matrices was observed, Shaw et al. (1995) found that the thorax components of variation were not significantly different between the base and small-thorax population, but bristle components were.

Of the comparative studies of genetic (co)variance matrices, only five have directly tested for the equality of these matrices (Shaw and Billington 1991; Plantenkamp and Shaw 1992; Brodie 1993; Paulsen 1996; Andersson, Shaw, and Widen unpubl. ms.). Of these, only Shaw and Billington
(1991) and Andersson, Shaw, and Widen (unpubl. ms.) used narrow-sense estimates of the genetic parameters. Shaw and Billington (1991) were only able to test for equality of variances because multivariate analyses gave poor convergence. Their results did suggest that the variances for flowering time differed between two sites. Andersson, Shaw, and Widen (unpubl. ms.) did find significant differences in both the variances and covariances for a number of traits, but did not compare entire $\mathbf{A}$ matrices. The remaining studies found no evidence for differences in the broad-sense genetic (co)variance matrices. Our results are consistent with these studies.

The power of the Wilkinson et al. (1990) study was likely enhanced by very large sample sizes, controlled laboratory conditions, and relatively large and constant heritabilities. Gromko (1995) and Bohren et al. (1966) suggested that when one trait is selected, as in the Wilkinson et al. (1990) study, the genetic variances of the traits genetically correlated with the selected trait should not change as much as the genetic covariances. Wilkinson et al. (1990) did not find differences in the variances, but did find differences in the covariances as predicted. Andersson, Shaw, and Widen (unpubl. ms.) also benefitted from heritabilities that were larger than those observed in this study. Thus, comparisons involving traits having at least moderate heritabilities have tended to have the power to detect differences.

A number of studies (e.g., Falconer 1960; Bell and McNary 1963; Wilkinson et al. 1990; Heath et al. 1995) utilized strong selection to detect significant differences in genetic (co)variance matrices. Such strong selection might lead to relatively large differences in such matrices. However, if selection is weak, or if means have differentiated by some other evolutionary process, then the genetic (co)variance matrices might not differ greatly. If the actual differences in most of the comparative studies are small, due to weak selection, then inordinately large experiments would be required to detect 
differences as significant. Similarly, the selection experiments might have been able to detect significant differences because the actual differences were sufficiently large.

Our results could reflect truly negligible differences among the $\mathbf{G}$ matrices of these populations. In that case, an implication is that selection has been weak and a sufficient number of loci underlie the traits examined to allow the (co)variance structure to be maintained by selection-mutation equilibrium (Lande 1976, 1980). Another possibility is that mutation is able to "restore" the original (co)variance structure following episodes of strong selection.

In a previous paper (Podolsky and Holtsford 1995), we provided evidence that historical selection had affected two continuous (IL1 and PW) and four discrete traits (WS, DB, $\mathrm{SC}$, and Pub) based on differences in the quantitative genetic population structure as estimated by $F_{\mathrm{ST}}$. These six traits exhibited estimates of $F_{\mathrm{ST}}$ that were significantly larger than allozyme estimates and were larger than $F_{S T}$-estimates from the other morphological traits, implying that selection had increased differentiation for these six morphological traits. The UPGMA clustering based on allozyme data did not reflect the geographical distribution of populations, leading Podolsky and Holtsford (1995) to suggest that selection has increased differentiation of the discrete traits between mountain ranges, but this selection has homogenized the discrete trait phenotypes within mountain ranges. The discrete traits examined are mainly floral traits that are similar to those in other species that have been found to be subject to some form of selection (e.g., Clegg and Epperson 1988; Rausher and Fry 1993). Jones $(1996 a, b)$ has shown that white cup in Clarkia gracilis (a similar phenotype to WS) has been subject to fertility selection. Our data are insufficient to determine the strength and duration of selection in C. dudleyana. Weak, long-term natural selection could result in divergence of means without causing the (co)variance structure to change drastically.

The results obtained from broad-sense comparisons of genetic variation might also yield different results than if narrow-sense variation were examined. In our study, we found consistent results for both the broad- and narrow-sense experiments, suggesting that both additive and nonadditive genetic variance show similar patterns of differentiation. However, differences between additive and nonadditive genetic (co)variance matrices might be expected. The presence of maternal effects could either increase or decrease the differentiation in the observed broad-sense genetic (co)variance matrices depending on the design of the experiment and the extent of maternal effects on covariances. For example, the common conditions experienced by the dams and sires of both populations used in the controlled crosses might be expressed as the progeny of the crosses exhibiting similar patterns of variation, overall. Such maternal effects could result in similar $\mathbf{G}$ matrices but different $\mathbf{A}$ matrices. Another potential for observing disparate results between broad- and narrow-sense estimates is that dominance components could be relatively large and more homogeneous than the additive genetic components. Traits affected more directly and more strongly by natural selection are expected to have relatively low heritabilities resulting from the erosion of additive genetic variance and the maintenance of relatively high levels of dominance variance (Wright 1929; Haldane 1932; Lerner 1954). Differences in the $\mathbf{A}$ matrices could therefore be masked when using broad-sense estimates.

While selection is one evolutionary process that could result in differentiation of genetic (co)variances, other processes might also play a role in this differentiation. Widen and Andersson (1993) examined $V_{A}$ for life-history and morphological traits in Senecio integrifolius in two populations that differed in numbers of individuals within the populations. The smaller and more patchy population displayed significant $V_{A}$ for more traits than did the larger population. Further, $V_{A}$ was shown to be significantly greater for many traits in the smaller population (Andersson, Shaw, and Widen, unpubl. ms.). These results led the authors to suggest that a combination of selection and spatial structure had been important in determining the differences in $\mathrm{V}_{\mathrm{A}}$.

Theory has shown that genetic drift can change A matrices (Avery and Hill 1977). Empirically, Bryant and Meffert (1993) and Bryant et al. (1986) have shown that founder events can increase genetic variances from an outbred population, and Bryant and Meffert (1996) suggested that the increased variances are due to epistatic interactions. This result of increased genetic variance following bottlenecks combined with the data of Widen and Andersson (1993) suggest that more theory is also needed for the spatial structure of quantitative genetic traits. Wright $(1943,1951)$ and Lande (1991) have shown that a structured population can maintain more genetic variation than a panmictic population if genetic variance is strictly additive, but no theory has explored the spatial structure of quantitative traits whose variation is at least partially nonadditive.

Despite clear morphological differentiation among populations, we fail to reject the null hypotheses that both additive and nonadditive genetic variances have remained relatively constant in $C$. dudleyana. Although the sometimes large observed differences in the additive (co)variances were not statistically significant, we cannot exclude the possibility that these differences are evolutionarily significant. Moreover, Shaw et al. (1995) showed that A matrices, as estimated in the laboratory, may appear constant, and indicated that selection response would likely differ from that predicted by laboratory estimates of $\mathbf{A}$ matrices. Selection response might also differ between populations due to the observed but statistically nonsignificant differences in nonadditive genetic variance and the potential for this variance to be converted to addititve genetic variance. Understanding the contribution of addititive and nonadditive genetic variance to differentiation of populations is an important area for further study.

\section{ACKNOWLEDGMENTS}

We thank N. Ellstrand, D. Reznick, A. Lukaszewski, and an anonymous reviewer for their valuable comments on the manuscript. G. Platenkamp provided many stimulating conversations for this work. We thank D. Larsen of the San Dimas Experimental Forest for allowing us access to the populations and for his assistance in finding these populations. This work was supported by a doctoral dissertation improvement grant, DEB-9119270, from the National Science Foundation to RHP. 


\section{Literature Cited}

ARNOLD, S. J. 1988. Quantitative genetics and selection in natural populations: microevolution of vertebral numbers in the garter snake, Thamnophis elegans. Pp. 619-636 in B. S. Weir, E. J. Eisen, M. M. Goodman, and G. Namkoong, eds. Proceedings of the second international conference on quantitative genetics. Sinauer, Sunderland, MA.

AVERY, P. J., AND W. G. HILl. 1977. Variability in genetic parameters among small populations. Genet. Res. (Cambridge) 29: 193-213.

Barton, N. H., AND M. Turelli. 1987. Adaptive landscapes, genetic distance and the evolution of quantitative characters. Genet. Res. 49:157-173.

Bell, A. E., AND H. W. MCNary. 1963. Genetic correlation and asymmetry of the correlated response to selection for increased bodyweight of Tribolium in two environments. P. 256 in J. Geerts, ed. Genetics today. Proceedings of the XI international congress of genetics. Vol. 1. Pergammon Press, MacMillan Co., New York.

Billington, H. L., A. M. Mortimer, AND T. MCNeilly. 1988. Divergence and genetic structure in adjacent grass populations. I. Quantitative genetics. Evolution 42:1267-1277.

Bohren, B. B., W. G. HiLl, AND A. RobertSon. 1966. Some observations on asymmetrical correlated responses to selection. Genet. Res. (Cambridge) 7:44-57.

BRODIE, E. D., III. 1993. Homogeneity of the genetic variancecovariance matrix for antipredator traits in two natural populations of the garter snake Thamnophis ordinoides. Evolution 47: 844-854.

Bryant, E. H., AND L. M. MefFert. 1993. The effect of serial founder-flush cycles on quantitative genetic variation in the housefly. Heredity 70:122-129.

- 1996. Nonadditive genetic structuring of morphometric variation in relation to a population bottleneck. Heredity 77 : $168-176$.

Bryant, E. H., S. A. McCommas, and L. M. Combs. 1986. The effect on an experimental bottleneck upon quantitative genetic variation in the housefly. Genetics 114:1191-1211.

ClegG, M. T., AND B. K. EpPerson. 1988. Natural selection of flower color polymorphisms in morning glory populations. Pp. 255-274 in L. D. Gottlieb and S. K. Jain, eds. Plant evoltuionary biology. Chapman and Hall, New York.

Comstock, R. E., AND H. F. RoBinson. 1948. The components of genetic variance in populations of biparental progenies and their use in estimating the average degree of dominance. Biometrics $4: 254-266$.

FALCONER, D. S. 1960. Selection for mice for growth on high and low planes of nutrition. Genet. Res. (Cambridge) 1:91-113.

- 1981. Introduction to quantitative genetics. 2d ed. Longman Scientific and Technical, New York.

FALCONER, D. S., AND T. F. C. MACKAY. 1996. Introduction to quantitative genetics. 4th ed. Longman Group Ltd., Essex, England.

Felsenstein, J. 1989. PHYLIP-phylogeny inference package. Vers. 3.2. Cladistics 5:164-166.

Freeman, M. F., AND J. W. TuKEY. 1950. Transformations related to the angular and square root. Ann. Math. Stat. 21:607-611.

Fujikoshi, Y. 1985. Selection of variables in discriminant analysis and canonical correlation analysis. Pp. 219-236 in P. R. Krishnaiah, ed. Multivariate analysis VI. Elsevier Science Publishers, New York.

GROMKO, M. H. 1995. Unpredictability of correlated response to selection: pleiotropy and sampling interact. Evolution 49:101109.

HaLdane, J. B. S. 1932. The causes of evolution. Longmans, Green and Co., New York.

Heath, S. C., G. Bulfield, R. Thompson, and P. D. Keightley. 1995. Rates of change of genetic parameters of body weight in selected mouse lines. Genet. Res. 66:19-25.

Hoffman, A. A., AND F. M. Cohan. 1987. Genetic divergence under uniform selection. III. Selection for knockdown resistance to ethanol in Drosophila pseudoobscura populations and their replicate lines. Heredity 58:425-433.

JONES, K. N. 1996a. Fertility selection on a discrete floral polymorphism in Clarkia (Onagraceae). Evolution 50:71-79.

- 1996b. Pollinator behavior and postpollination reproductive success in alternative floral phenotypes of Clarkia gracilis (Onagraceae). Int. J. Plant Sci. 157:733-738.

KoHN, L. A. P., AND W. R. ATCHLEY. 1988. How similar are genetic correlation structures: data from mice and rats. Evolution 42: 467-481.

LANDE, R. 1976. The maintenance of genetic variability by mutation in a polygenic character with linked loci. Genet. Res. 26 221-234.

- 1979. Quantitative genetic analysis of multivariate evolution, applied to brain: body size allometry. Evolution 33:402416.

1980. The genetic covariance between characters maintained by pleiotropic mutations. Genetics $94: 203-215$.

. 1991. Isolation by distance in a quantitative trait. Genetics 128:443-452.

. 1992. Neutral theory of quantitative genetic variance in an island model with local extinction and colonization. Evolution 46:381-389.

LERNER, I. M. 1954. Genetic homeostasis. Oliver and Boyd, Edinburgh, Scotland.

LEWIS, H. 1962. Catastrophic selection as a factor in speciation. Evolution 16:257-271.

Lewis, H., AND M. E. LEwIs. 1955. The genus Clarkia. Univ. Calif. Publ. Bot. 20:241-392.

Lewis, H., AND P. H. Raven. 1958. Rapid evolution in Clarkia. Evolution 12:319-336.

LOFSVOLD, D. 1986. Quantitative genetics of morphological differentiation in Peromyscus. I. Tests of the homogeneity of genetic covariance structure among species and subspecies. Evolution 40:559-573.

-1 1988. Quantitative genetics of morphological differentiation in Peromyscus. II. Analysis of selection and drift. Evolution 42:54-67.

MCCulloch, C. E. 1994. Maximum likelihood variance components estimation for binary data. J. Am. Stat. Assoc. 89:330 335.

NAGYLAKI, T. 1994. Geographical variation in a quantitative character. Genetics 136:361-381.

PAUlSEN, S. M. 1996. Quantitative genetics of the wing color patterns in the buckeye butterfly (Precis coenia and Precis evarete): evidence against the constancy of G. Evolution 50:1585-1597.

Platenkamp, G. A. J., AND R. G. Shaw. 1992. Environmental and genetic constraints on adaptive population differentiation in $A n$ thoxanthum odoratum. Evolution 46:341-352.

Podolsky, R. H., AND T. P. HoltsFord. 1995. Population structure of morphological traits in Clarkia dudleyana. I. Comparison of $F_{\text {ST }}$ between allozymes and morphological traits. Genetics 140: 733-744.

Price, T. D., AND P. R. Grant. 1985. The evolution of ontogeny in Darwin's finches: a quantitative genetic approach. Am. Nat. 124:169-188.

Price, T. D., P. R. Grant, and P. T. Boag. 1984. Genetic changes in the morphological differentiation of Darwin's ground finches. Pp. 49-66 in K. Wohrmann and V. Loeschcke, eds. Population biology and evolution. Springer-Verlag, Berlin.

Rausher, M. D., AND J. D. FRY. 1993. Effects of a locus affecting floral pigmentation in Ipomoea purpurea on female fitness components. Genetics 134:1237-1247.

SAS InSTITUTE. 1985. SAS user's guide: statistics. Vers. 5 ed. SAS Institute, Inc., Cary, NC.

Shaw, F. H., AND C. J. Geyer. 1997. Estimation and testing in constrained covariance component models. Biometrika 84:95102.

Shaw, F. H., R. G. Shaw, G. S. Wilkinson, and M. Turelli. 1995. Changes in genetic variances and covariances: $G$ whiz! Evolution 49:1260-1267.

SHAw, R. G. 1987. Maximum-likelihood approaches applied to 
quantitative genetics of natural populations. Evolution 41:812826.

-1 1991. The comparison of quantitative genetic parameters between populations. Evolution 45:143-151.

Shaw, R. G., AND H. L. Billington. 1991. Comparison of variance components between two populations of Holcus lanatus: a reanalysis. Evolution 45:1287-1289.

Shaw, R. G., AND F. H. Shaw. 1992. Quercus: programs for quantitative genetic analysis using maximum likelihood. Published electronically on the Internet, available directly from the authors or via anonymous ftp from ftp.bio.indiana.edu; directory path biology/quantgen/quercus.

SNEATH, P. H. A., AND R. R. SoKal. 1973. Numerical taxonomy: the principles and practice of numerical classification. Freeman, San Francisco, CA.

TURELl, M. 1988a. Phenotypic evolution, constant covariances, and the maintenance of additive variance. Evolution 42:13421347.

1988b. Population genetic models for polygenic variation and evolution. Pp. 601-628 in B. S. Weir, E. J. Eisen, M. M. Goodman, and G. Namkoong, eds. Proceedings of the second international conference on quantitative genetics. Sinauer, Sunderland, MA.

Turelli, M., J. H. Gillespie, AND R. Lande. 1988. Rates tests for selection on quantitative characters during macroevolution and microevolution. Evolution 42:1085-1089.

WIDEN, B., AND S. ANDERSSON. 1993. Quantitative genetics of lifehistory and morphology in a rare plant, Senecio integrifolius. Heredity 70:503-514.

Wilkinson, G. S., K. Fowler, And L. Partridge. 1990. Resistance of genetic correlation structure to directional selection in Drosophila melanogaster. Evolution 44:1990-2003.

WRIGHT, S. 1929. Fisher's theory of dominance. Am. Nat. 63:274279.

- 1943. Isolation by distance. Genetics 28:114-138. 1951. The genetical structure of populations. Ann. Eugen. 15:323-354.
APPENDIX

TABLE A1. Collection sites for the 11 populations of Clarkia dudleyana.

\begin{tabular}{|c|c|}
\hline $\begin{array}{l}\text { Popula- } \\
\text { tion }\end{array}$ & Collection information \\
\hline 118 & $\begin{array}{l}\text { San Bernardino Co., SSE of Yucaipa; T2S R2W S8 } \\
\text { SW } 1 / 4\end{array}$ \\
\hline 119 & $\begin{array}{l}\text { Los Angeles Co., NNE of Glendora; T1N R9W S11 } \\
\text { SW1/4 }\end{array}$ \\
\hline $\begin{array}{l}123 \\
124\end{array}$ & $\begin{array}{l}\text { San Bernardino Co., N of Upland; T1N R8W S13 W1/2 } \\
\text { Los Angeles Co., N of San Dimas, Tanbark Flats Exper- } \\
\text { iment Station in the San Dimas Experimental Forest; } \\
\text { T1N R8W S6 NW1/4 }\end{array}$ \\
\hline 128 & $\begin{array}{l}\text { San Bernardino Co., N of Fontana; T2N R6W S25 } \\
\text { SW } 1 / 4\end{array}$ \\
\hline 129 & Riverside Co., N of Banning; T4S R1E S10 NE1/4 \\
\hline 130 & $\begin{array}{l}\text { San Bernardino Co., SW of Running Springs; T1N } \\
\text { R3W S1 W1/2 }\end{array}$ \\
\hline 132 & $\begin{array}{l}\text { San Bernardino Co., NE of San Bernardino; T1N R3W } \\
\text { S13 SE1/4 }\end{array}$ \\
\hline $\begin{array}{l}133 \\
135\end{array}$ & $\begin{array}{l}\text { Los Angeles Co., N of Glendora; T2N R8W } \\
\text { Riverside Co., NW of Idylwild: T4S R2E S6 SW1/4 }\end{array}$ \\
\hline 136 & $\begin{array}{l}\text { Los Angeles Co., N of San Dimas; TIN R9W S12 } \\
\text { SW1/4 }\end{array}$ \\
\hline
\end{tabular}

TABLE A2. Means and standard deviations for the continuous morphological traits by population measured in mm. Means are on the first line and standard deviations appear on the second. Sample sizes appear in parentheses.

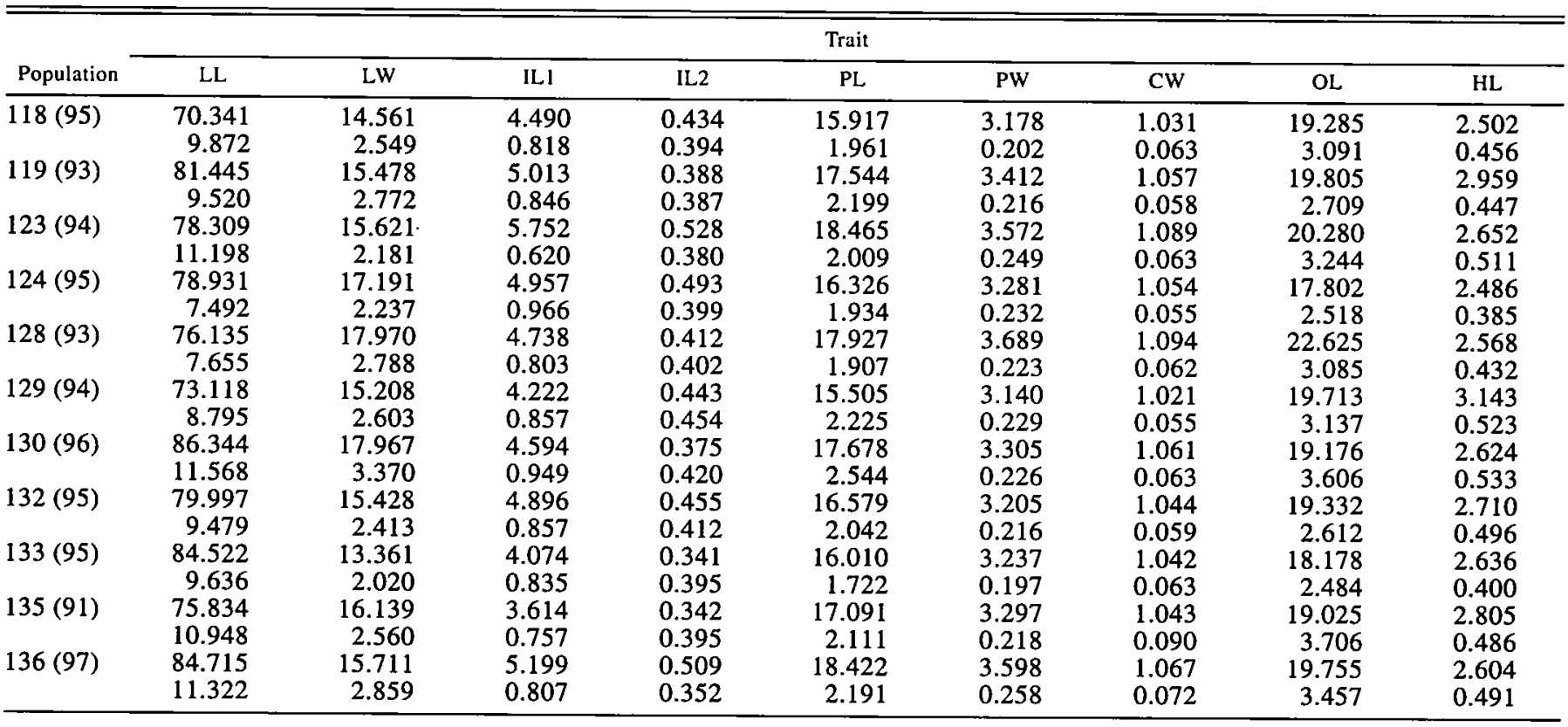


ROBERT H. PODOLSKY ET AL.

TABLE A3. Frequencies of the discrete traits by population.

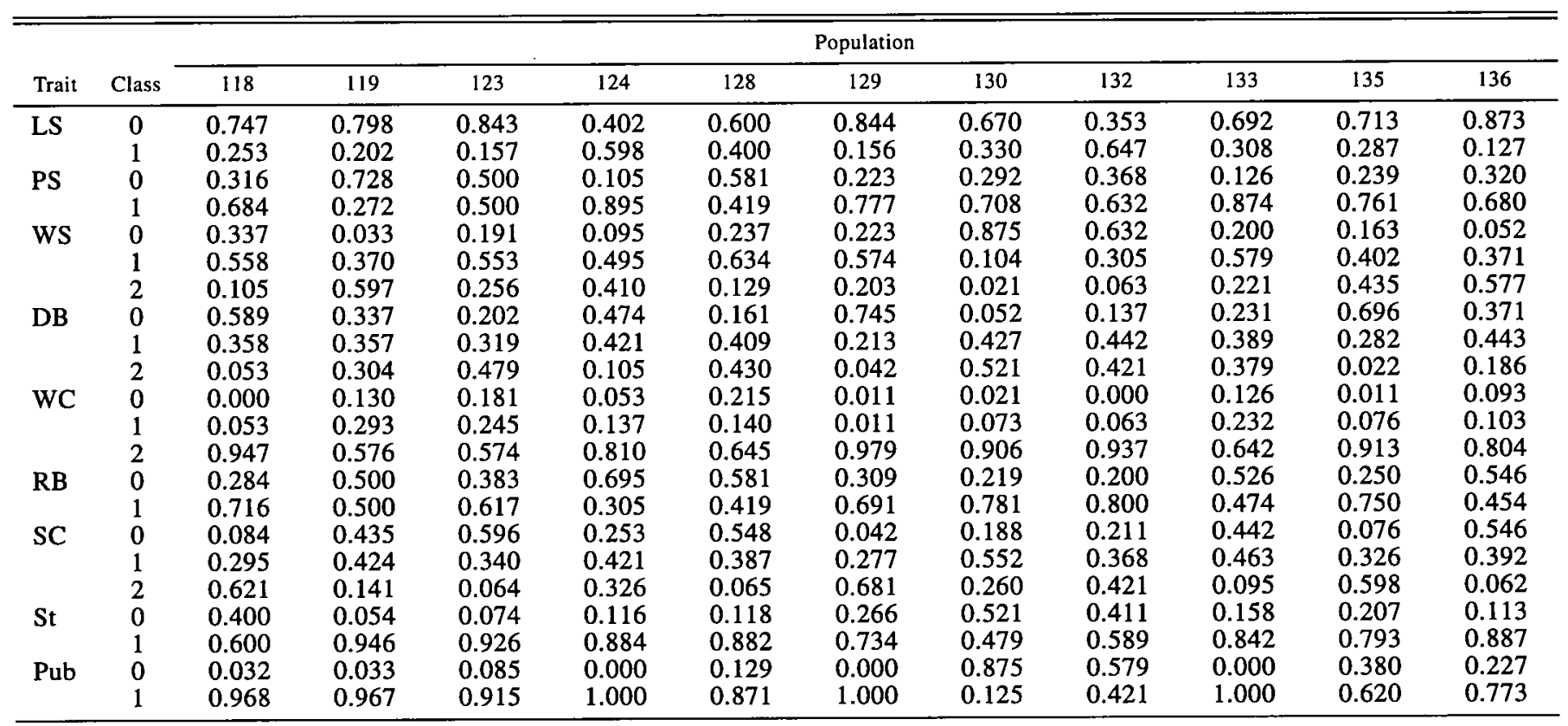

